\title{
Ratio of high-sensitivity troponin to creatine kinase-MB in takotsubo syndrome
}

\author{
Charles Pirlet ${ }^{*, 1}$, Luc Pierard ${ }^{1}$, Victor Legrand ${ }^{1}$, Olivier Gach ${ }^{1}$ \\ CHU de Liège, avenue de l'hôpital, 1, Liège 4000, Belgium
}

\section{A R T I C L E I N F O}

\section{Article history:}

Received 18 April 2017

Received in revised form 21 May 2017

Accepted 29 May 2017

Available online $\mathrm{xxxx}$

\section{Keywords:}

Takotsubo

High-sensitivity troponin T

Biomarkers

Creatine kinase-MB

\begin{abstract}
A B S T R A C T
Background: Takotsubo syndrome (TT) and myocardial infarction (MI) share numerous similarities in clinical presentation, ECG modifications and biomarker elevation. We sought to determine whether the ratio of highsensitivity cardiac troponin $\mathrm{T}$ (hs-TnT) to the myocardial fraction of creatine kinase (CKMB) could be a potent discriminator between TT and MI patients.

Methods: We separately present analysis of data from retrospective files and prospectively recruited patients presenting with TT ( 35 retrospective and 42 prospective), NSTEMI ( 48 retrospective and 75 prospective) and STEMI (20 retrospective and 39 prospective). We compared ratios of hs-TnT to CKMB on admission to the hospital between TT, NSTEMI and STEMI patients. Receiver operating characteristic (ROC) curves were analysed to determine optimal cut-off values.

Results: On admission, hs-TnT/CKMB ratio was significantly higher in TT patients than in NSTEMI and STEMI patients in both the retrospective phase (median and interquartile range, TT 0.024 [0.018-0.047] vs NSTEMI 0.009 [0.006-0.022], $\mathrm{p}<0.0001$; TT vs STEMI 0.011 [0.006-0.016], $\mathrm{p}=0.0002$ ) and the prospective cohort (median and interquartile range, TT 0.032 [0.018-0.040] vs NSTEMI 0.009 [0.006-0.015], $\mathrm{p}<0.0001$; TT vs STEMI 0.009 [0.005-0.017], $\mathrm{p}<0.0001$ ). A cut-off hs-TnT/CKMB ratio of 0.015 distinguished TT from MI with a sensitivity of $85.7 \%$ and a specificity of $67.6 \%$ (AUC $0.796 ; 95 \% \mathrm{CI}$ : $0.71-0.89$ ) in the retrospective phase. In the prospective phase, a ratio of 0.017 distinguished TT from MI with a sensitivity of $83.3 \%$ and a specificity of 78.1\% (AUC 0.88; 95\%CI: 0.83-0.94).

Conclusion: hs-TnT/CKMB ratio is a novel, readily available parameter that could be used alongside clinical risk scores, other biomarkers and ECG findings to discriminate between TT and MI.
\end{abstract}

(c) 2017 Elsevier B.V. All rights reserved.

\section{Introduction}

Takotsubo syndrome (TT) is an increasingly diagnosed condition [1-3] and our understanding of its clinical profile has evolved. Straying away from the initial description [4,5] of ST elevation following an acute psychological stress, different electrocardiographic (ECG) presentations and other triggers, if any, are now more commonly reported [6]. Furthermore, bystander coronary artery disease is present in up to $15 \%$ of reported cases [6-8]. Thus, clinicians are frequently confronted with challenging cases where diagnosis is equivocal especially when imaging of the left ventricle is performed late in the clinical course. Moreover, many authors now suggest that invasive imaging may not be necessary in patients presenting without ST segment elevation $[9,10]$. In this setting, much research has been conducted to find discriminatory markers between acute coronary syndromes, especially ST-segment elevation

\footnotetext{
* Corresponding author.

E-mail address: cpirlet@student.ulg.ac.be (C. Pirlet).

1 This author takes responsibility for all aspects of the reliability and freedom from bias of the data presented and their discussed interpretation.
}

myocardial infarction (STEMI), and TT as appropriate diagnosis has important implications with regards to management and prognosis.

This study was inspired by a simple clinical observation: we know that patients with TT present with smaller elevations in troponin and creatine kinase than STEMI $[11,12]$. However, as observed by others, the elevation in creatine kinase [7,13], more specifically its myocardial fraction (CKMB), is smaller in comparison to the elevation in troponin than in myocardial infarction (MI). In other words, the ratio of troponin to CKMB seems higher in TT than in MI. Therefore, after gathering our retrospective data, we designed a single centre prospective study to test whether the ratio of high-sensitivity troponin $\mathrm{T}$ (hs-TnT) to CKMB could be used as a discriminatory marker between these two entities. In this report, we present both our retrospective and prospective data.

\section{Methods}

This study was conducted at the Liège University Hospital (CHU de Liège), Belgium, which accepts referrals for acute coronary syndromes from other regional hospitals. 
TT patients had to have been hospitalized for the diagnosis of TT and had to have undergone coronary angiography and left ventriculography. Diagnosis of TT was established according to recently revised criteria [6]: i). Transient regional wall motion abnormalities (WMA) extending beyond a single epicardial vascular distribution. ii). Absence of culprit coronary artery disease (thrombus, severe stenosis, spontaneous dissection) or other pathological conditions to explain the pattern of temporary left ventricular dysfunction. iii). New and reversible ECG abnormalities. iv). Positive but relatively small elevation in cardiac troponin. Follow-up at least three months after the index hospitalization confirming normalisation of wall motion abnormalities and left ventricular systolic function was obtained through review of medical files and by telephone contact with the cardiologist of the patient. Cardiac magnetic resonance imaging (MRI) during the index hospitalization to exclude myocarditis was performed at the discretion of the treating physician. In accordance with recent literature, many patients benefited from cardiac MRI three months after the index event to confirm regression of WMA and to exclude the presence of late gadolinium enhancement due to other causes such as MI or myocarditis. However, as this study was purely observational, cardiac MRI was not part of the study protocol.

Concerning MI patients, this study was limited to type $1 \mathrm{MI}$ patients [14] confirmed by coronary angiography presenting within $36 \mathrm{~h}$ of symptom onset. These patients were classified as STEMI and NSTEMI patients according to their initial ECG presentation. Only those with biomarker elevations prior to percutaneous coronary intervention (PCI) were considered and laboratory data after PCI was discarded in order to observe only the natural course of these cardiac biomarkers.

Exclusion criteria included significant kidney disease (defined arbitrarily in accordance with other similar studies [10] by a glomerular filtration rate (GFR) inferior to $35 \mathrm{~mL} / \mathrm{min} / 1.73 \mathrm{~m}^{2}$ according to the MDRD formula) and cardiac/muscle biomarker elevation due to other causes.

Retrospective data were extracted from the digital medical files from November 2011 to April 2014. High-sensitivity troponin T assays were available at Liège University Hospital as from November 2011. All female patients with type $1 \mathrm{MI}$ without ST segment elevation were deemed suitable for inclusion. To limit the NSTEMI and STEMI sample sizes, the samples of male patients were arbitrarily limited to a random population totalling $25 \%$ of the NSTEMI and STEMI cohorts. This seemed logical as the incidence of TT is believed to be higher in women. Data from all eligible patients were collected.

Prospective inclusion of patients started in April 2014 and ended in December 2016. To limit the NSTEMI sample size without introducing selection bias, these patients were recruited only amongst those present in the coronary unit on one specific day of the week.

The study was approved by the institutional ethics review committee and complied with the principles of the Helsinki Declaration. All prospectively recruited patients provided informed consent.

hs-TnT and CKMB were simultaneously measured on admission and then every $6 \mathrm{~h}$ within the first $24 \mathrm{~h}$ according to the institutional protocol. For all patients, relevant data included age at the index event, gender, smoking status, beta-blocker therapy, GFR and up to five simultaneous hs-TnT and CKMB measurements. For TT patients, ECG presentation, history of vascular or coronary disease, ballooning pattern and recurrence were also recorded. In the prospective phase, NT-proBNP was also measured on admission. Logically, markers of cardiac necrosis prior to $\mathrm{PCI}$ were more seldom available in STEMI patients except when blood was drawn before primary $\mathrm{PCI}$ or in patients ineligible to $\mathrm{PCI}$.

Analyses for hs-TnT were performed with TnT-hs (Elecsys ${ }^{\circledR}$, Roche diagnostics, Vilvoorde, Belgium; upper limit of normal (ULN) $14 \mathrm{ng} / \mathrm{L}$ ). NT-proBNP was measured with ProBNP II (Cobas ${ }^{\circledR}$, Roche diagnostics, Vilvoorde, Belgium; ULN 156 ng/L). CKMB were analysed with CK-MB (Cobas ${ }^{\circledR}$, Roche diagnostics, Vilvoorde, Belgium, ULN $6.2 \mu \mathrm{g} / \mathrm{L}$ ).

In both studies (retrospective and prospective), the primary analysis was a significant difference in the ratio of hs-TnT to CKMB between TT, NSTEMI and STEMI patients for the first blood analysis recorded. The same ratio was also analysed amongst the three groups at the sample time when hs-TnT was at its peak. Values of troponin and CKMB were compared between groups on admission and at the sample time when hs-TnT was at its peak. In the prospective population, NT-proBNP and the ratio of NT-proBNP to peak hs-TnT were also studied.

Continuous measures are described with the use of mean and standard deviation (SD) for parametric data and median and interquartile range (IQR) for non-parametric data. Categorical data were summarized as frequency and percentages. Data was checked for normal distribution with the Shapiro-Wilk test and homogeneity of variance was confirmed with Bartlett's test/Fisher's test where appropriate. A log transformation (base 10) was applied to laboratory measurements that did not respect a normal distribution. The Pearson chi-square test was used for categorical variables. Between group comparisons of continuous variables were performed with a one-way analysis of variance (ANOVA) followed by Tukey's post-hoc test if the null hypothesis was rejected. Welch's ANOVA was used when the assumption of equal variance was not met despite log transformation. Results were compared using the unpaired Student $t$-test when only two groups (TT and MI) were compared such as for the NT-proBNP measurements. Receiver operator characteristic (ROC) curves were calculated and optimal cut-off values (Youden's J statistic) to distinguish TT from MI were determined. The area under the curve (AUC) and the sensitivity and specificity at the optimal cutoff point are reported. A 2 -sided probability value $<0.05$ was considered statistically significant. All statistical analyses were performed with $\mathrm{R}$ version 3.3.1 (The R Foundation for Statistical Computing, Vienna, Austria) and the EZR package.

All the authors were implicated in the design of the study and review of the manuscript. The authors vouch for the integrity of the data.

\section{Results}

From November 2011 to May 2014, 35 TT patients fulfilled the inclusion criteria for the retrospective phase of the study. These patients were compared with 48 NSTEMI patients and 20 STEMI patients. Thereafter, 47 TT patients were prospectively enrolled to be compared with 75 NSTEMI patients and 39 STEMI patients who were enrolled over the same period. There was no interruption between the retrospective and the prospective phases of the study. The study ended on December 31, 2016.

In the prospective phase, two TT patients could not be included because of incomplete biomarker profiles prior to referral for coronary angiography. Lastly, three patients were excluded because of late gadolinium enhancement on cardiac MRI that was suggestive of another disease process than TT (MI in two cases and myocarditis in the third).

Population characteristics are presented in Table 1 . In line with the usual gender distribution of TT, there were more females in the TT populations. This was also the case in the retrospective MI populations, reflecting the pre-specified sample bias to limit study population. In both the retrospective and the prospective trials, age, smoking, beta-blocker use and kidney function were similar albeit, as expected, more active smokers in the MI populations.

In both trials, apical ballooning was the predominant type of WMA. Recurrences were rare. A substantial proportion of TT patients had concomitant coronary artery disease. 6 patients in the retrospective phase had a cardiac MRI in the course of their follow-up. In the prospective phase, $18(42.9 \%)$ patients had cardiac MRI. 4 patients (5.2\%) could not be contacted despite repeated efforts and $5(6.5 \%)$ patients died soon after the index event before follow-up imaging was performed.

Medians and interquartile ranges for hs-TnT, CKMB and the ratio of hs-TnT to CKMB are displayed in Table 2 and Fig. 1. In both phases of the trial, the ratios of hs-TnT to CKMB, on admission and at the peak troponin value, proved to be statistically different between TT patients and the MI subgroups. However, hs-TnT was not significantly different between groups in our population both on admission and at its peak value, in the two phases of the study whereas statistically significant 
Table 1

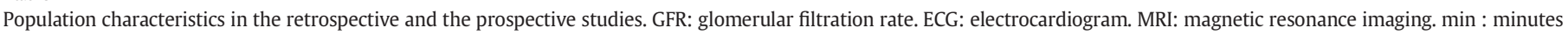
(median).

\begin{tabular}{|c|c|c|c|c|}
\hline \multirow[b]{2}{*}{ Retrospective - no. } & \multirow{2}{*}{$\begin{array}{l}\text { Takotsubo } \\
35 \\
\end{array}$} & \multirow{2}{*}{$\frac{\text { NSTEMI }}{48}$} & \multirow{2}{*}{$\frac{\text { STEMI }}{20}$} & \multirow[t]{2}{*}{ p-Value } \\
\hline & & & & \\
\hline Age - years (mean $\pm S D)$ & $69.8 \pm 10$ & $69.3 \pm 14.2$ & $68.4 \pm 15.2$ & 0.923 \\
\hline Female sex - no. (\%) & $32(91.4)$ & $35(72.9)$ & $15(75)$ & 0.1 \\
\hline Tobacco use & & & & 0.78 \\
\hline Active smokers - no. (\%) & $7(20)$ & $13(27.1)$ & $6(30)$ & \\
\hline Past smokers - no. (\%) & $10(28.6)$ & $12(25)$ & $3(15)$ & \\
\hline Beta-blocker use - no. (\%) & $12(34.4)$ & $25(52.1)$ & $10(50)$ & 0.25 \\
\hline Kidney disease & & & & 0.888 \\
\hline GFR $45-60 \mathrm{~mL} / \mathrm{min} / 1.73 \mathrm{~m}^{2}-$ no. (\%) & $5(14.3)$ & $9(18.8)$ & $5(25)$ & \\
\hline GFR $35-45 \mathrm{~mL} / \mathrm{min} / 1.73 \mathrm{~m}^{2}-$ no. (\%) & $3(8.6)$ & $4(8.3)$ & $1(5)$ & \\
\hline Normal ECG - no. (\%) & $6(17.1)$ & & & \\
\hline ST elevation - no. (\%) & $10(28.6)$ & & & \\
\hline Q wave - no. (\%) & $1(2.9)$ & & & \\
\hline Other modifications - no. (\%) & $18(51.4)$ & & & \\
\hline Apical - no. (\%) & $28(80)$ & & & \\
\hline Midventricular - no. (\%) & $7(20)$ & & & \\
\hline Basal - no. (\%) & $0(0)$ & & & \\
\hline Recurrence - no. (\%) & $1(2.9)$ & & & \\
\hline Cardiac MRI - no. (\%) & $6(17.1)$ & & & \\
\hline Coronary disease - no. (\%) & $8(22.9)$ & & & \\
\hline Vascular disease (non-coronary) - no. (\%) & $3(8.6)$ & & & \\
\hline Delay from symptoms to biomarker - min & 1451 & 462 & 215 & 0.0002 \\
\hline Prospective - no. & 42 & 75 & 39 & \\
\hline Age - years $($ mean $\pm S D)$ & $69.3 \pm 12.3$ & $65.2 \pm 14$ & $64.9 \pm 12.7$ & 0.23 \\
\hline Female sex - no. (\%) & $38(90.5)$ & $26(34.7)$ & $11(28.2)$ & $<0.0001$ \\
\hline Smoker & & & & 0.799 \\
\hline Current - no. (\%) & $12(28.6)$ & $30(40)$ & $14(35.9)$ & \\
\hline Former - no. (\%) & $12(28.6)$ & $18(24)$ & $11(28.2)$ & \\
\hline Beta-blocker use - no. (\%) & $19(45.2)$ & $30(40)$ & $18(46.2)$ & 0.771 \\
\hline Kidney disease & & & & 0.108 \\
\hline GFR $45-60 \mathrm{~mL} / \mathrm{min} / 1.73 \mathrm{~m}^{2}-$ no. (\%) & 5 (11.9) & $14(18.7)$ & $4(10.3)$ & \\
\hline GFR $35-45 \mathrm{~mL} / \mathrm{min} / 1.73 \mathrm{~m}^{2}-$ no. (\%) & $5(11.9)$ & $1(1.3)$ & $2(5.1)$ & \\
\hline Normal ECG - no. (\%) & $10(23.8)$ & & & \\
\hline ST elevation - no. (\%) & $9(21.4)$ & & & \\
\hline Q wave - no. (\%) & $7(16.7)$ & & & \\
\hline Other modifications - no. (\%) & $16(38.1)$ & & & \\
\hline Apical - no. (\%) & $28(66.7)$ & & & \\
\hline Midventricular - no. (\%) & $13(31)$ & & & \\
\hline Basal - no. (\%) & $1(2.3)$ & & & \\
\hline Recurrence - no. (\%) & $2(4.7)$ & & & \\
\hline Cardiac MRI - no. (\%) & $18(42.9)$ & & & \\
\hline Coronary disease - no. (\%) & $7(16.7)$ & & & \\
\hline Vascular disease (non-coronary) - no. (\%) & $4(9.5)$ & & & \\
\hline Delay from symptoms to biomarker - min & 350.5 & 351.5 & 331.5 & 0.72 \\
\hline
\end{tabular}

Table 2

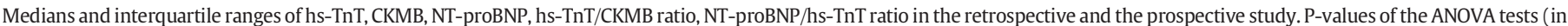

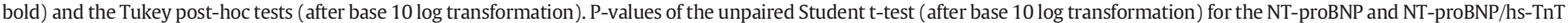
ratio. CKMB: myocardial fraction of creatine kinase. hs-TnT: high-sensitivity troponin T. ANOVA: analysis of variance.

\begin{tabular}{|c|c|c|c|c|}
\hline & Retrospective & $\mathrm{p}$ value & Prospective & $\mathrm{p}$ value \\
\hline \multirow[t]{3}{*}{ hs-TnT (ng/L) on admission } & & 0.28 & & 0.23 \\
\hline & TT 317 [91.5-433] vs NSTEMI 218.5 [43.3-333.5] & NA & TT 198 [137.3-265.5] vs NSTEMI 158 [62-313] & NA \\
\hline & TT 317 [91.5-433] vs STEMI 122.5 [70.5-248.5] & NA & TT 198 [137.3-265.5] vs STEMI 248 [119-700] & NA \\
\hline \multirow[t]{3}{*}{ CKMB $(\mu \mathrm{g} / \mathrm{L})$ on admission } & & 0.0321 & & $<0.0001$ \\
\hline & TT 7 [3.8-12.2] vs NSTEMI 10.1 [5.2-22.7] & NS & TT 6.9 [5.1-10] vs NSTEMI 14.3 [6-37.7] & 0.0005 \\
\hline & TT 7 [3.8-12.2] vs STEMI 14.6 [7.3-30.9] & 0.0371 & TT 6.9 [5.1-10] vs STEMI 23.7 [8.5-89.2] & $<0.0001$ \\
\hline \multirow[t]{3}{*}{ Peak hs-TnT (ng/L) } & & 0.0599 & & 0.2158 \\
\hline & TT 362 [175.5-699] vs NSTEMI 354.5 [222.8-705.5] & NA & TT 341.5 [209.5-508.8] vs NSTEMI 525 [291-868] & NA \\
\hline & TT 362 [175.5-699] vs STEMI 158 [81.8-711] & NA & TT 341.5 [209.5-508.8] vs STEMI 399 [186-1732] & NA \\
\hline \multirow[t]{3}{*}{ CKMB $(\mu \mathrm{g} / \mathrm{L})$ with peak hs-TnT } & & 0.0074 & & $<0.0001$ \\
\hline & TT 10.6 [5-17.9] vs NSTEMI 15.9 [9.7-42.5] & 0.0068 & TT 10.4 [6.9-16.6] vs NSTEMI 35.6 [16.7-59.7] & 0.0001 \\
\hline & TT 10.6 [5-17.9] vs STEMI 16.7 [8.7-39.9] & NS & TT 10.4 [6.9-16.6] vs STEMI 51.8 [19.8-119.2] & 0.0001 \\
\hline \multirow[t]{2}{*}{ hs-TnT/CKMB on admission } & & $<0.0001$ & & $<0.0001$ \\
\hline & TT 0.024 [0.018-0.047] vs NSTEMI 0.009 [0.006-0.022] & $<0.0001$ & TT 0.032 [0.018-0.04] vs NSTEMI 0.009 [0.006-0.015] & $<0.0001$ \\
\hline \multirow[t]{3}{*}{ hs-TnT/CKMB for peak hs-TnT } & TT 0.024 [0.018-0.047] vs STEMI 0.011 [0.006-0.016] & $\begin{array}{l}0.0002 \\
<0.0001\end{array}$ & TT 0.032 [0.018-0.04] vs STEMI 0.009 [0.005-0.017] & $\begin{array}{l}<0.0001 \\
<0.0001\end{array}$ \\
\hline & TT 0.029 [0.023-0.045] vs NSTEMI 0.018 [0.011-0.033] & 0.002 & TT 0.033 [0.021-0.046] vs NSTEMI 0.018 [0.009-0.025] & 0.0001 \\
\hline & TT $0.029[0.023-0.045]$ vs STEMI 0.011 [0.007-0.016] & $<0.001$ & TT $0.033[0.021-0.046]$ vs STEMI 0.010 [0.007-0.018] & 0.0001 \\
\hline NT-proBNP (ng/L) & & & TT 3719 [1825-8171.5] vs MI 691.5 [277.3-1804.5] & $<0.0001$ \\
\hline NT-proBNP/peak hs-TnT & & & TT $7.7[3.6-36.5]$ vs MI $1.2[0.2-4.5]$ & $<0.0001$ \\
\hline
\end{tabular}




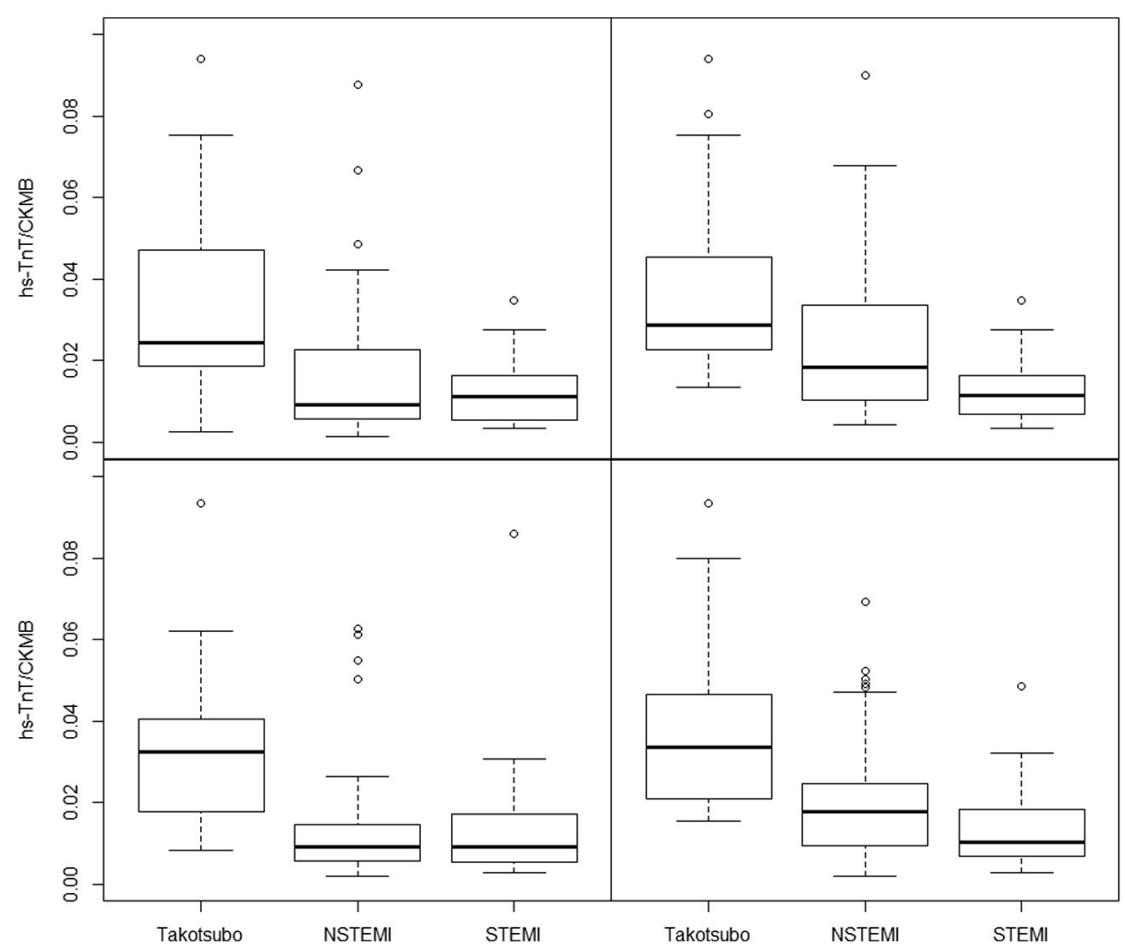

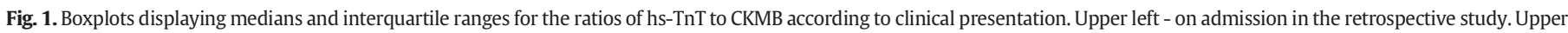

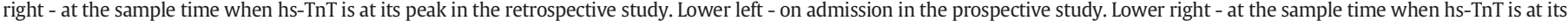
peak in the prospective study. CKMB: myocardial fraction of creatine kinase. hs-TnT: high-sensitivity troponin T.

differences in CKMB values were observed on admission and at the corresponding peak troponin sample times. As previously reported with conventional troponin $[9,10,13,15]$, the ratio of NT-proBNP to hs-TnT proved to be significantly different (Table 2) between TT and MI patients (NT-proBNP available in 23 and 26 patients respectively). ROC curves (Fig. 2) and their respective AUC show that the optimal hs-TnT/CKMB cut-off to distinguish TT from MI is on admission with a value of 0.015 in the retrospective cohort (sensitivity $85.7 \%$, specificity of $67.6 \%$, AUC $0.796 ; 95 \% \mathrm{CI}: 0.71-0.89$ ) and, in the prospective phase, a ratio of 0.017 (sensitivity of $83.3 \%$, specificity $78.1 \%$, AUC $0.88 ; 95 \%$ CI: 0.83-0.94). Surprisingly, the ROC curve of the ratio of NT-proBNP to hs-TnT indicated a lower AUC $(0.77 ; 95 \% \mathrm{CI}$ : 0.63-0.91) than for the ratio of hs-TnT/CKMB in the prospective study (Fig. 2).

\section{Discussion}

These two studies were a proof-of-concept work which served to demonstrate our hypothesis that the ratio of hs-TnT troponin to CKMB could be used as an early indicator of TT. Indeed, the ratio of hs-TnT to CKMB was significantly higher in TT patients than in NSTEMI and STEMI patients on admission and later in the course of the event, when troponin reached its peak value. In addition, at a threshold of 0.015 , in the retrospective phase, and 0.017 , in the prospective phase, this ratio yields reasonable sensitivity and specificity.

We believe the diagnoses of TT in both populations to be sufficiently supported by follow-up imaging and, in a significant proportion of patients, cardiac MRI. This point is of critical importance in studies seeking to establish discriminatory parameters between TT and MI patients. Furthermore, clinical characteristics (age, sex, ballooning pattern, presence of bystander coronary artery disease) of our TT populations are comparable to those of larger trials [7]. Our findings also suggest an increase (12/year in the retrospective phase versus 15.8/year in the prospective phase) in the diagnosis of TT as previously described $[1,3]$.

This was an observational clinical study. Notwithstanding, it may give some insights into the mechanisms behind cell death in TT. Indeed, our data suggest that cell death in TT is different to that of MI. This is also supported by histological studies which show contraction band necrosis similar to that observed in myocardial stunning following subarachnoid haemorrhage or in ischemia-reperfusion syndromes [16]. This is in contrast to the coagulation necrosis observed in MI. Moreover, fundamental studies on rats by Paur et al. [17] suggest that catecholamine toxicity may be the dominant process. In that case, the kinetics of cardiac biomarker leakage in TT may be different to those of cardiomyocyte necrosis in MI.

In contrast to previous reports concerning conventional troponin $[7,11]$, hs-TnT values were not significantly different between groups, even at peak values. We believe that the progression of hs-TnT and CKMB in our MI populations may have been blunted by the fact that only blood samples drawn prior to PCI were taken into account. Thus, peak troponin values are probably underestimated, especially in the STEMI groups.

It is well known that CKMB values drop earlier than troponin values after MI [18]. Therefore, the longer the delay after the beginning of the event, especially after peak CKMB values, the higher the ratio of hs-TnT to CKMB. This may mitigate the difference in hs-TnT ratios between TT and MI. With this concept in mind, we took great care not to include patients with MI presenting late $(>36 \mathrm{~h}$ ) after symptom onset. The lesser AUC values at the peak troponin time compared to admission are consistent with this hypothesis. Our intent in recording serial hs-TnT and CKMB samples in individual patients was to capture these values at varying time lapses after the onset of the event. This is of importance as the delay between the onset of TT and admission is often unknown and variable.

We performed post-hoc analyses of the delays from symptom onset to the first biomarker sample in both our populations. As specified above, the onset of symptoms in TT is sometimes unclear so data were not always available. Nevertheless, in the retrospective phase, TT patients presented significantly later than MI patients. This difference in presentation delays begs the question whether the statistically significant differences between our MI populations and TT populations could simply be explained by different times from symptom onset to biomarker analysis. Thus, we compared the tertile of early TT presenters with 


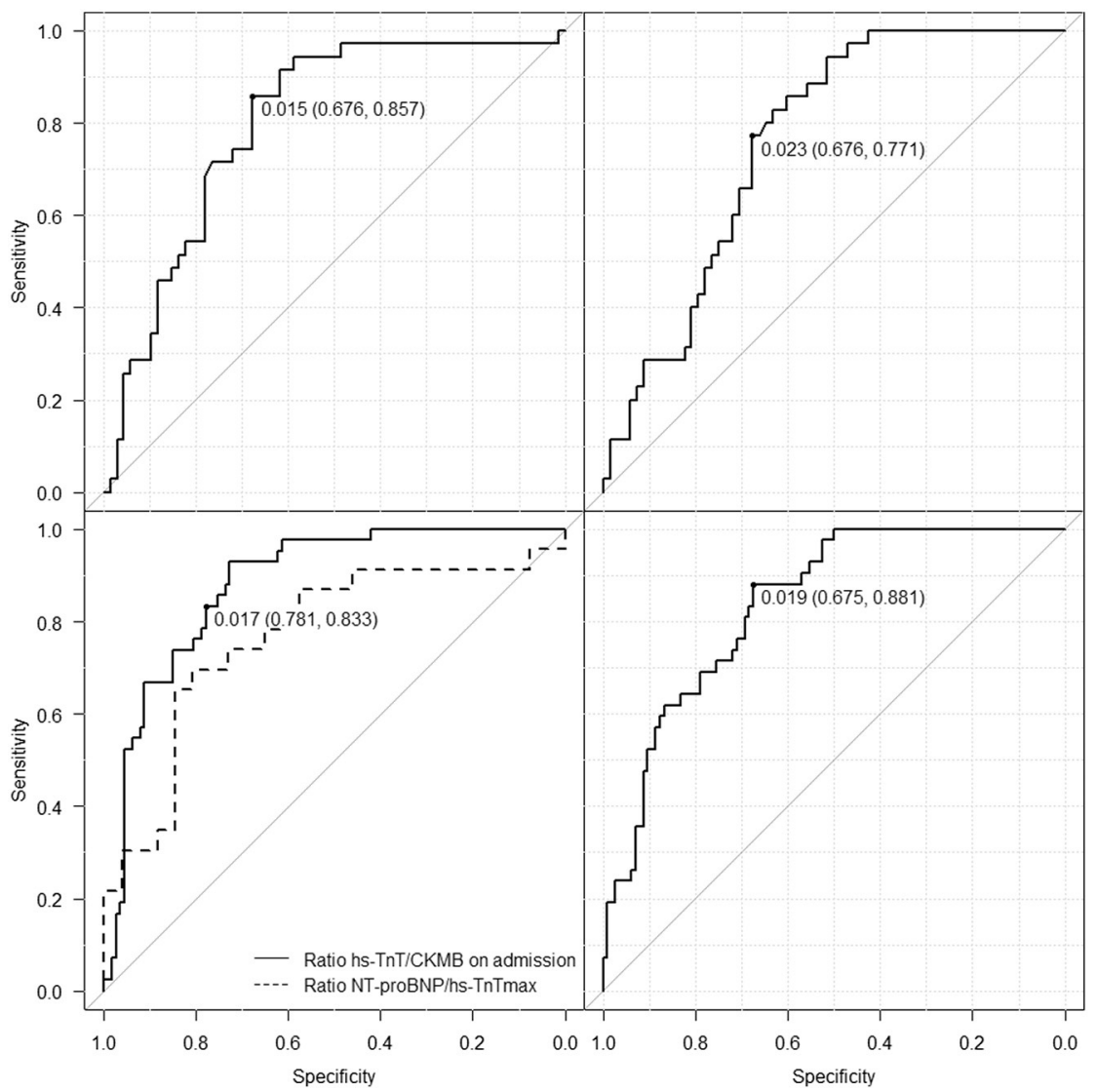

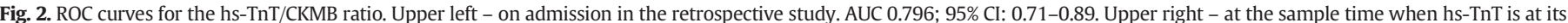

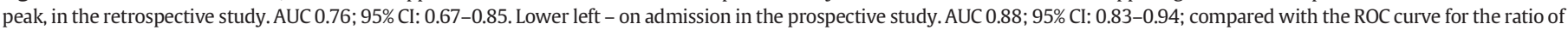

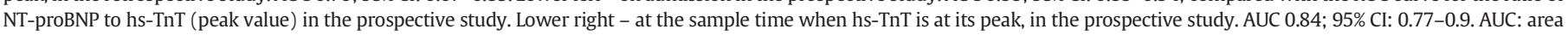
under the curve. CI: confidence interval. CKMB: myocardial fraction of creatine kinase. hs-TnT: high-sensitivity troponin T. ROC: receiver operator characteristic.

the NSTEMI and STEMI cohorts. The ratio of hs-TnT to CKMB was still significantly higher in this selected population than in myocardial infarction patients. Then, we compared the hs-TnT/CKMB ratio amongst the three tertiles of takotsubo patients (early, intermediate and late presenters). There were no statistical differences in hs-TnT/CKMB ratio between these tertiles. Therefore, we consider our observations to hold true regardless of the delay to presentation in TT patients. There were no significant differences in the interval between symptom onset and the first biomarker sample in the prospective phase. This discrepancy between the retrospective phase and the prospective phase is thought to be related to slightly different methodologies, unavailable data for TT patients with no cardiovascular symptoms and a high prevalence of late presenting STEMI patients in this part of the study.

There are some other notable differences in results between the retrospective study and the prospective study. These discrepancies are probably related to random sampling in these relatively small populations.

Graphically, the interquartile range for NSTEMI seems to be wider at peak values than on admission. This is probably due to the fact that NSTEMI is a heterogeneous subset of disease encompassing mild forms that would probably have been classified as unstable angina with conventional troponin assays and more severe acute coronary syndromes with significant myocardial necrosis. In this regard, we acknowledge that NSTEMI patients with minor hs-TnT and no CKMB elevation displayed high hs-TnT/CKMB ratios. Perhaps, this parameter should only be calculated in patients with CKMB above the ULN.

Our trial is the first to our knowledge to study hs-TnT in TT patients. This is of importance as high-sensitivity troponin assays are currently widely used in Europe as recommended in current guidelines of the European Society of Cardiology [19]. Surprisingly, the revised criteria for TT include elevation of troponin measured with a conventional assay [6].
The clinical presentations of TT and MI bear numerous similarities. These two entities cannot be differentiated by clinical presentation or classic diagnostic tools such as ECG and baseline laboratory data. Epidemiology and risk factor profiles may be different but there is much overlap to the point that bystander non culprit coronary artery disease is now no longer an exclusion criterion in the diagnosis of TT. In the setting of ST segment elevation, most will agree that the risk of denying timely reperfusion therapy through primary PCI outweighs the risk of occasionally performing invasive imaging in TT. However, in NSTEMI patients, clinicians are often confronted with difficult clinical scenarios where echocardiography suggests TT and the decision to perform coronary angiography may be in question. Thus, adjunctive discriminators are an interesting concept.

To this end, several parameters have shown reasonable diagnostic performance. Of these, the ratio of BNP or NT-proBNP to troponin has been extensively studied $[9,10,13,15]$. Of note, two of these studies only compared TT with STEMI patients in whom management would not be altered on the basis of biomarkers which are often available only after coronary angiography. Looi et al. [20] showed the utility of analysing two ECG markers, namely the number of leads with T-wave inversion and peak T-wave inversion $>3 \mathrm{~mm}$, on the second day after admission. The product of peak troponin I to ejection fraction has also proved to be a potent discriminator (accuracy 91\%) with STEMI patients [21]. Desmet et al. [22] showed that the presence of an "apical nipple sign" on left ventriculography provided $100 \%$ specificity for the diagnosis of TT (in comparison to STEMI patients). More recently, Ghadri et al. [23] published the Inter-TAK Diagnostic Score which is based on the largest TT cohort ever published. This score assembles clinical and ECG markers.

Further study is required to confirm the diagnostic performance of the ratio of hs-TnT to CKMB. The clinical utility behind this parameter 
resides in the fact that it is readily available, with baseline blood analyses, within the first hour of management. Secondly, the Inter-TAK score does not integrate laboratory parameters. We believe that adding the ratio of hs-TnT to CKMB and/or the ratio of BNP to troponin to such a clinical risk score could enhance its diagnostic performance. Thirdly, for different reasons, coronary angiography is sometimes performed late during the clinical course, after resolution of typical WMA. In these patients, especially those with mild coronary artery disease, diagnosis and future management are difficult and the ratio of hs-TnT to CKMB may then offer a retrospective diagnostic utility.

The magnitude of troponin and/or CKMB could also be of value in ruling out TT. We observed that no TT patient exceeded the thresholds of $1770 \mathrm{ng} / \mathrm{L}$ and $50.7 \mu \mathrm{g} / \mathrm{L}$ for hs-TnT and CKMB respectively. This last component could also be used in a diagnostic algorithm.

Three patients were excluded because cardiac MRI showed late gadolinium enhancement compatible with MI (in two cases) or myocarditis. Like others [6-8], we believe that cardiac MRI should become a standard of care in the management of TT patients. Once again, correct diagnosis is important for the management of individual patients but it also has implications with regards to further research on TT.

\section{Study limitations}

Asides from the fact that this was a single centre trial studying two relatively small cohorts, the major limitation of this study was the selection of the NSTEMI and STEMI groups in both phases of the trial. As with any study that requires a convenience sample of patients, the selection process may introduce bias into the results.

The second limitation regards ascertaining the diagnosis of TT. Few trials have a flawless methodology in this regard. In our study, performing systematic cardiac MRI in the prospective phase was not possible and most patients did not have cardiac MRI in the retrospective phase. Therefore, we resorted to checking follow-up imaging although this may not exclude patients who experienced other reversible phenomena that could mimic TT.

Thirdly, no calculations were performed to assess the prospective sample size.

Fourth, strictly speaking, we did not observe the natural course of biomarker profiles as most patients in all groups benefited from medical therapy.

Fifth, we studied a limited population with regards to racial distribution. All TT patients were Caucasians.

Lastly, we did not make any attempt to match the different study populations because their respective characteristics have been recognized as inherently different.

\section{Conclusion}

The two observational studies show a significant difference in the ratio of hs-TnT to CKMB between TT patients and both NSTEMI and STEMI patients. As such, this readily available parameter yields reasonable sensitivity and specificity. Integrating it in a diagnostic score with other biomarkers may be relevant.

Further research in larger prospective trials is necessary to confirm our findings and to determine the diagnostic performance of this parameter.

\section{Conflict of interest}

No conflict of interest to declare.

\section{Acknowledgments}

This work was supported by the fonds d'investissement de recherche scientifique (FIRS) of the Liège University Hospital (CHU de Liège).

\section{References}

[1] R. Khera, K. Light-McGroary, F. Zahr, P.A. Horwitz, S. Girotra, Trends in hospitalization for takotsubo cardiomyopathy in the United States, Am. Heart J. 172 (2016) 53-63.

[2] B. Redfors, R. Vedad, O. Angeras, et al., Mortality in takotsubo syndrome is similar to mortality in myocardial infarction - a report from the SWEDEHEART registry, Int. J. Cardiol. 185 (2015) 282-289.

[3] K. Murugiah, Y. Wang, N.R. Desai, et al., Trends in short- and long-term outcomes for takotsubo cardiomyopathy among medicare fee-for-service beneficiaries, 2007 to 2012, JACC Heart Fail. 4 (2016) 197-205.

[4] K. Dote, H. Sato, H. Tateishi, T. Uchida, M. Ishihara, Myocardial stunning due to simultaneous multivessel coronary spasms: a review of 5 cases, J. Cardiol. 21 (1991) 203-214.

[5] K. Tsuchihashi, K. Ueshima, T. Uchida, et al., Transient left ventricular apical ballooning without coronary artery stenosis: a novel heart syndrome mimicking acute myocardial infarction. Angina pectoris-myocardial infarction investigations in Japan, J. Am. Coll. Cardiol. 38 (2001) 11-18.

[6] A.R. Lyon, E. Bossone, B. Schneider, et al., Current state of knowledge on takotsubo syndrome: a position statement from the Taskforce on Takotsubo Syndrome of the Heart Failure Association of the European Society of Cardiology, Eur. J. Heart Fail. 18 (2016) 8-27.

[7] C. Templin, J.R. Ghadri, J. Diekmann, et al., Clinical features and outcomes of takotsubo (stress) cardiomyopathy, N. Engl. J. Med. 373 (2015) 929-938.

[8] F. Ugo, M. Iannaccone, F. D'Ascenzo, et al., Prevalence and characterization of bystander coronary artery disease in tako-tsubo cardiomyopathy using a multiimaging approach, Int. J. Cardiol. 209 (2016) 51-53.

[9] M.S. Randhawa, A.S. Dhillon, H.C. Taylor, Z. Sun, M.Y. Desai, Diagnostic utility of cardiac biomarkers in discriminating takotsubo cardiomyopathy from acute myocardial infarction, J. Card. Fail. 20 (2014) 2-8.

[10] G.M. Frohlich, B. Schoch, F. Schmid, et al., Takotsubo cardiomyopathy has a unique cardiac biomarker profile: NT-proBNP/myoglobin and NT-proBNP/troponin T ratios for the differential diagnosis of acute coronary syndromes and stress induced cardiomyopathy, Int. J. Cardiol. 154 (2012) 328-332.

[11] G. Parodi, P.S. Del, N. Carrabba, et al., Incidence, clinical findings, and outcome of women with left ventricular apical ballooning syndrome, Am. J. Cardiol. 99 (2007) 182-185.

[12] T.M. Pilgrim, T.R. Wyss, Takotsubo cardiomyopathy or transient left ventricular apical ballooning syndrome: a systematic review, Int. J. Cardiol. 124 (2008) 283-292.

[13] M. Madhavan, B.A. Borlaug, A. Lerman, C.S. Rihal, A. Prasad, Stress hormone and circulating biomarker profile of apical ballooning syndrome (takotsubo cardiomyopathy): insights into the clinical significance of B-type natriuretic peptide and troponin levels, Heart 95 (2009) 1436-1441.

[14] K. Thygesen, J.S. Alpert, A.S. Jaffe, et al., Third universal definition of myocardial infarction, Eur. Heart J. 33 (2012) 2551-2567.

[15] M. Budnik, J. Kochanowski, R. Piatkowski, et al., Simple markers can distinguish takotsubo cardiomyopathy from ST segment elevation myocardial infarction, Int. J. Cardiol. 219 (2016) 417-420.

[16] I.S. Wittstein, D.R. Thiemann, J.A. Lima, et al., Neurohumoral features of myocardial stunning due to sudden emotional stress, N. Engl. J. Med. 352 (2005) 539-548.

[17] H. Paur, P.T. Wright, M.B. Sikkel, et al., High levels of circulating epinephrine trigger apical cardiodepression in a beta2-adrenergic receptor/Gi-dependent manner: a new model of Takotsubo cardiomyopathy, Circulation 126 (2012) 697-706.

[18] A.H. Wu, F.S. Apple, W.B. Gibler, R.L. Jesse, M.M. Warshaw, R. Valdes Jr., National Academy of Clinical Biochemistry standards of laboratory practice: recommendations for the use of cardiac markers in coronary artery diseases, Clin. Chem. 45 (1999) 1104-1121

[19] M. Roffi, C. Patrono, J.P. Collet, et al., 2015 ESC guidelines for the management of acute coronary syndromes in patients presenting without persistent ST-segment elevation: Task Force for the Management of Acute Coronary Syndromes in Patients Presenting without Persistent ST-Segment Elevation of the European Society of Cardiology (ESC), Eur. Heart J. 37 (2016) 267-315.

[20] J.L. Looi, C.W. Wong, M. Lee, A. Khan, M. Webster, A.J. Kerr, Usefulness of ECG to differentiate takotsubo cardiomyopathy from acute coronary syndrome, Int. J. Cardiol. 199 (2015) 132-140.

[21] F.O. Nascimento, S. Yang, M. Larrauri-Reyes, et al., Usefulness of the troponinejection fraction product to differentiate stress cardiomyopathy from ST-segment elevation myocardial infarction, Am. J. Cardiol. 113 (2014) 429-433.

[22] W. Desmet, J. Bennett, B. Ferdinande, et al., The apical nipple sign: a useful tool for discriminating between anterior infarction and transient left ventricular ballooning syndrome, Eur. Heart J. Acute Cardiovasc. Care 3 (2014) 264-267.

[23] J.R. Ghadri, V.L. Cammann, S. Jurisic, et al., A novel clinical score (InterTAK diagnostic score) to differentiate takotsubo syndrome from acute coronary syndrome: results from the international takotsubo registry, Eur. J. Heart Fail. (2016). 